\title{
Evaluación de las actividades realizadas mediante la metodología de Aprendizaje Basado en Problemas
}

\author{
Ramón M. Fernández-Domene ${ }^{\mathrm{a}}$, Rita Sánchez-Tovar ${ }^{\mathrm{b}}$, Gemma Roselló-Márquez ${ }^{\mathrm{a}}$, Patricia Batista- \\ Grau $^{\mathrm{a}}$ y José García-Antón ${ }^{\mathrm{a}}$ \\ ${ }^{a}$ Departmento de Ingeniería Química y Nuclear, Universitat Politècnica de València, Camino de Vera s/n, 46022 \\ Valencia, Spain \\ ${ }^{\mathrm{b}}$ Departmento de Ingeniería Química, Universitat de València, Av de les Universitats, s/n, 46100 Burjassot, Spain
}

Abstract

The Problem-Based Learning (ABP) methodology requires an evaluation approach in which a series of specific and transversal competencies are evaluated. This evaluation must be formative allowing students to identify and correct mistakes in time. Therefore, it is necessary to have tools in order to evaluate suitably the learning outcomes and established competencies. In the subject of Corrosion which belongs to the second course of the Master of Chemical Engineering, the ABP has been evaluated through a report to be handed after each ABP session, where the main conclusions are collected after the cooperative resolution of the proposed problem and, on the other hand, by means of the notes taken by the teachers and students (co-assessment and selfassessment), after the oral presentation of the results and conclusions of the practice. In this work, for each of these evaluation activities, specific rubrics have been developed. From the different evaluation activities, it can be affirmed that the rubrics used are effective for the evaluation of the ABP methodology activities. In addition, it was possible to verify that the students have reached the learning results and it was also possible to detect the corrosion issues that pose the greatest difficulty to the students.

Keywords: problem-based learning; self-assessment; co-assessment; rubric; corrosion

\section{Resumen}

La metodología de Aprendizaje Basado en Problemas (ABP) requiere un enfoque de evaluación en el que se evalúen una serie de competencias específicas y transversales. Esta evaluación debe de tener un carácter formativo que permita a los estudiantes identificar y corregir los errores a tiempo. Por lo tanto, es necesario disponer de herramientas para poder evaluar apropiadamente los resultados de aprendizaje y las competencias establecidas. En la asignatura de Corrosión de segundo de Máster de Ingeniería Química, el ABP se ha evaluado a través de un informe a entregar tras cada sesión de ABP, en donde se recogen las principales conclusiones tras la resolución cooperativa del problema planteado y, por otra parte, mediante las notas tomadas por el profesorado y por el alumnado (coevaluación y autoevaluación), tras la exposición oral de los resultados y conclusiones de la práctica de campo. En este trabajo, para cada una de estas actividades de evaluación, se han desarrollado unas rúbricas especificas. A partir de las distintas actividades de evaluación se puede afirmar que las rúbricas empleadas son efectivas a la hora de evaluar la metodología ABP. Además, se ha podido comprobar que el alumnado ha alcanzado los resultados de aprendizaje y se han podido detectar los temas de corrosión que plantean mayor dificultad al alumnado.

Palabras clave: aprendizaje basado en problemas; autoevaluación; co-evaluación; rúbrica; corrosión 


\section{Introducción}

El proceso educativo cuenta con dos figuras principales como son el docente y el estudiante, cuyos roles son muy diferentes en la enseñanza tradicional, en donde el primero tiene un peso más importante, mientras que el segundo adopta un comportamiento más pasivo. Sin embargo, existen otras técnicas de aprendizaje como es el aprendizaje basado en problemas (ABP), la cual se origina durante los sesenta en la Escuela de Medicina de la Universidad de McMaster, en Ontario, para aproximar a los estudiantes al ambiente que encontrarán en su vida profesional mediante la resolución de escenarios problemáticos (Nuñez Lopez et al, 2017). En la metodología ABP se pretende realizar una correcta formulación de diferentes casos prácticos que promueven al estudiante a reconocer, investigar y asimilar conceptos y fundamentos que requerirán para la resolución de los problemas. En este nuevo ambiente de formación, ambas figuras no deben ser pasivas y por tanto, se deben comprometer a mostrar una actitud dinámica, siendo el primer paso estar predispuesto al cambio. Con esto se quiere conseguir dejar atrás la idea de que aprender es memorizar, ya que aunque es parte del proceso no es el principio más importante de la técnica (Shaw et al, 2006). Además, el uso de la tecnología en el modelo ABP es un factor importante ya que se emplea como apoyo en la organización de las clases, favorece el contacto o interacción entre ambas figuras y facilita la realización del seguimiento del proceso (Ausín et al, 2016).

Por tanto, el ABP consiste en la búsqueda y posterior propuesta de soluciones a problemas que ocurren en la vida real con el objetivo de aportar conocimientos y a su vez estimular y crear un diálogo que facilite la evaluación crítica y objetiva de las diferentes alternativas. En el ABP, el docente se convierte en un facilitador de la información en vez de ser un simple transmisor del conocimiento, para intentar generar un pensamiento reflexivo en los estudiantes (Wyness y Dalton, 2018).

Esta metodología aporta muchos beneficios en el desarrollo tanto personal como académico de los alumnos. A continuación se detallan los que cobran más importancia.

- Aumentar la motivación. Con esta metodología se consigue proponer problemas que sean de actualidad así como llamativos e importantes. Además, se ha demostrado que se logra una mayor motivación del estudiante ya que aumenta la asistencia a clase, promueve una mayor participación y un mayor interés por las tareas propuestas.

- Revisión y reflexión. El ABP promueve una actitud basada en la continua revisión y retroalimentación de las soluciones aportadas. Con esto se consigue que los alumnos asuman que cometer errores no es algo malo y que cada trabajo realizado se debe revisar antes de darlo por finalizado. Por tanto, con el ABP se crean muchas oportunidades en las que reflexionar y revisar el proyecto ya que los alumnos pueden criticarse de manera constructiva entre ellos además de recibir la opinión de los docentes, consiguiéndose así unos estudiantes más independientes y más críticos tanto consigo mismo como con los compañeros.

- Preparar a los estudiantes para el futuro. Esta metodología desarrolla en los estudiantes la mejora de las aptitudes tales como la colaboración y trabajo en equipo, la planificación del trabajo, la toma de decisiones y pensamiento crítico (Toledo Morales y Sánchez García, 2018).

- Aumentar la autoestima. Para los alumnos siempre resulta motivador y satisfactorio alcanzar resultados y metas que tienen aplicabilidad en la vida real.

- Hablar en público: La metodología de ABP plantea a los alumnos la opción de realizar una pequeña presentación de los resultados obtenidos a los compañeros. Con ello se consigue que los estudiantes puedan contar todas las limitaciones con las que se han encontrado y los problemas 
que les han surgido a lo largo del proyecto, mejorando conjuntamente la habilidad de hablar en público (Kassem, 2018).

No obstante, la implementación del ABP aun presenta algunas cuestiones importantes como es la viabilidad de la implementación de la metodología en todos los niveles escolares y si es igual de beneficioso usar ABP con algunas simplificaciones de los problemas reales, ya que en ciertos niveles son necesarias debido al gran nivel de dificultad que tiene su resolución. A pesar de estas reticencias esta metodología se ha implementado en los diferentes niveles: primaria, secundaria, bachillerato y universidad. Por tanto, el objetivo principal de esta investigación es evaluar la contribución de esta iniciativa docente desarrollada en el aula a través de los resultados de aprendizaje logrados durante el proceso de enseñanza.

\section{Plan de trabajo o metodología}

\section{Contexto de la asignatura y resultados de aprendizaje}

La metodología activa de ABP se ha implementado en la asignatura "Corrosión", que se imparte actualmente en el Máster Universitario en Ingeniería Química ( $2^{\circ}$ curso) ofertado por la Universitat Politècnica de València. La asignatura consta de 4.5 créditos en total. El objetivo de esta asignatura es el de capacitar al alumno para identificar los diferentes procesos de corrosión, conocer las técnicas experimentales para su estudio y saber aplicar diversas técnicas de protección a los problemas industriales y científicos específicos. La asignatura se estructura en cuatro unidades didácticas. La metodología de ABP se implementará especialmente en las últimas tres unidades didácticas, que abordan las celdas de corrosión, las técnicas de protección contra la corrosión y las estrategias de diseño que cualquier profesional debería de seguir para minimizar o eliminar este fenómeno indeseado que puede generar graves pérdidas económicas y humanas, así como las aplicaciones industriales y de proceso.

Para las dos unidades didácticas mencionadas en el párrafo anterior, se pretende que el alumnado alcance los siguientes resultados de aprendizaje:

1. Distinguir entre los distintos fenómenos y celdas de corrosión que pueden aparecer en un sistema real.

2. Comparar entre distintos diseños de equipos o instalaciones para determinar cuál es el mejor con el objetivo de minimizar la corrosión.

3. Explicar las diferentes técnicas de protección frente a la corrosión existentes y adaptarlas para casos reales particulares.

4. Explicar y justificar, argumentando adecuadamente, las opciones elegidas a la hora de resolver problemas reales.

5. Explicar de forma sintética, clara y sin ambigüedades las principales conclusiones alcanzadas tras la resolución de cada problema.

Las dos unidades didácticas se desarrollarán en 7 sesiones, con lo que se trabajará con un total de 7 problemas reales, que las y los estudiantes deberán resolver en clase. El número de alumnos matriculados ha sido de 10 . 


\section{Actividades de enseñanza/aprendizaje}

Las actividades de enseñanza/aprendizaje que se han planteado para lograr un adecuado desarrollo de la metodología de ABP se han llevado a cabo en el aula (salvo que se mencione lo contrario). Estas actividades se describen a continuación:

1. Resolución de problemas empleando la metodología de ABP: las y los estudiantes, organizados en parejas o grupos de pequeño tamaño, debieron partir de los conocimientos previos de que disponían y buscar de forma cooperativa la información necesaria para identificar el problema de corrosión que estaba teniendo lugar en cada caso y, además, plantear una o varias soluciones al mismo. El personal docente tuvo en todo momento un papel secundario, ejerciendo de guía del alumnado cuando fue necesario. Una vez resuelto el problema, el alumnado entregó la solución al personal docente, con el fin de llevar a cabo un feedback continuo del correcto funcionamiento de la metodología ABP. Los diferentes problemas están diseñados para que puedan realizarse en el aula en el tiempo dado.

2. Corrección del problema de la sesión anterior y feedback: tras entregar el informe con la resolución del problema planteado, el profesor o profesora repartió los informes del problema de la sesión anterior corregidos y realizó una pequeña presentación que recogía los principales puntos de dicho problema. De esta manera, el alumnado sabía de forma continua cuál fue la calificación de su trabajo y en qué había podido fallar, proporcionándole un feedback semanal muy importante para continuar con su aprendizaje de forma adecuada.

3. Práctica de campo: una de las sesiones de clase se invirtió en realizar una práctica de campo. En dicha práctica, el alumnado hizo una inspección de los edificios e instalaciones de la Universitat Politècnica de València para detectar problemas de corrosión que afectaran a infraestructuras de la universidad y que supusieran pérdidas materiales y pudieran poner en riesgo la seguridad de las personas que forman parte de la comunidad universitaria. Tras esta inspección, redactaron un informe en donde se presentaron estos problemas de corrosión y se plantearon posibles soluciones para corregir y evitar estos problemas en el futuro, así como unas conclusiones finales.

4. Presentación de la práctica de campo: en otra sesión de aula, cada grupo realizó una exposición oral de los resultados y conclusiones principales a los que llegaron tras realizar la práctica de campo. Tras la exposición de cada grupo, se abrió un pequeño debate para que el resto de grupos dieran su opinión sobre los problemas presentados por sus compañeras y compañeros.

\section{Actividades de evaluación}

Las metodologías activas de enseñanza/aprendizaje, como el ABP, requieren un enfoque de evaluación diferente, en el que se evalúen una serie de competencias específicas y transversales. Además, esta evaluación debe de tener un carácter formativo, lo que permite a los estudiantes identificar y corregir los errores a tiempo. Para ello, es necesario disponer de herramientas para poder evaluar con garantías los resultados de aprendizaje y las competencias establecidas. Para esta asignatura, el ABP se ha evaluado usando diferentes actividades de evaluación: (1) informe a entregar tras cada sesión, en donde se recogen 
las principales conclusiones tras la resolución cooperativa del problema planteado; (2) notas tomadas por el profesorado y por el alumnado (coevaluación y autoevaluación) tras la exposición oral de los resultados y conclusiones de la práctica de campo. Para cada una de estas actividades de evaluación se han desarrollado unas rúbricas que se presentarán más adelante. A continuación se describen con más detalle las dos actividades de evaluación de ABP empleadas en esta asignatura:

1. Entrega del informe grupal del ABP para cada problema: tras finalizar cada sesión de ABP, el alumnado entregó un informe en donde se tenía que recoger, de forma sintética, el análisis y la resolución del problema planteado. No sólo se valoró la solución final, sino también el camino seguido hasta llegar a esa conclusión. Se realizó una evaluación individual a partir del informe grupal mediante una rúbrica, con una nota numérica (0-10) ordenada en cuatro bloques (suspenso, aprobado, notable y sobresaliente) (Tabla 1). Puesto que la evaluación tuvo en todo momento un carácter formativo, los alumnos conocieron sus calificaciones a lo largo de toda la asignatura, recibiendo de esta manera información constante sobre sus progresos y sus carencias. Esto les ayudó a autocorregirse y a continuar desarrollando sus habilidades y competencias.

2. Exposición oral tras la práctica de campo: se evaluó el trabajo de todo el grupo a partir de la presentación oral sobre los resultados y conclusiones principales obtenidas durante la práctica de campo. Esta evaluación la realizó el profesorado y también el alumnado (coevaluación y autoevaluación) rellenando una rúbrica sencilla en la que se valoran en una escala numérica del 1 al 4 una serie de ítems relacionados con los resultados y conclusiones presentadas (Tabla 2). Se evaluó la capacidad del alumnado para argumentar y defender sus posturas, así como la capacidad para consensuar durante los debates. 
Tabla 1. Rúbrica para la actividad de evaluación (1)

\begin{tabular}{|c|c|c|c|c|}
\hline \multirow[b]{2}{*}{ Resultados de aprendizaje } & \multicolumn{4}{|c|}{ Nivel de competencia } \\
\hline & $\begin{array}{l}\text { Sobresaliente } \\
9-10\end{array}$ & $\begin{array}{c}\text { Notable } \\
7-8\end{array}$ & $\begin{array}{c}\text { Aprobado } \\
5-6\end{array}$ & $\begin{array}{c}\text { Suspenso } \\
0-4\end{array}$ \\
\hline $\begin{array}{l}\text { Distinguir entre los distintos fenómenos y celdas de } \\
\text { corrosión que pueden aparecer en un sistema real }\end{array}$ & $\begin{array}{l}\text { Distingue e identifica todos los } \\
\text { fenómenos y celdas de corrosión } \\
\text { que aparecen en el sistema bajo } \\
\text { estudio }\end{array}$ & $\begin{array}{l}\text { Distingue e identifica la mayoría } \\
\text { de los fenómenos y celdas de } \\
\text { corrosión que aparecen en el } \\
\text { sistema bajo estudio }\end{array}$ & $\begin{array}{l}\text { Distingue e identifica algunos de } \\
\text { los fenómenos y celdas de } \\
\text { corrosión que aparecen en el } \\
\text { sistema bajo estudio }\end{array}$ & $\begin{array}{l}\text { No identifica los fenómenos y } \\
\text { celdas de corrosión que } \\
\text { aparecen en el sistema bajo } \\
\text { estudio }\end{array}$ \\
\hline $\begin{array}{l}\text { Comparar entre distintos diseños de equipos o } \\
\text { instalaciones para determinar cuál es el mejor con el } \\
\text { objetivo de minimizar la corrosión }\end{array}$ & $\begin{array}{l}\text { Compara entre distintos diseños de } \\
\text { forma completa y precisa y } \\
\text { determina correctamente el mejor } \\
\text { de ellos }\end{array}$ & $\begin{array}{l}\text { Compara entre distintos diseños de } \\
\text { forma notable y determina } \\
\text { correctamente el mejor de ellos }\end{array}$ & $\begin{array}{l}\text { Compara entre distintos diseños } \\
\text { de forma imprecisa y no llega a } \\
\text { determina correctamente el mejor } \\
\text { de ellos }\end{array}$ & $\begin{array}{l}\text { No compara entre distintos } \\
\text { diseños y no determina el } \\
\text { mejor de ellos }\end{array}$ \\
\hline $\begin{array}{l}\text { Explicar las diferentes técnicas de protección frente a la } \\
\text { corrosión existentes y adaptarlas para casos reales } \\
\text { particulares }\end{array}$ & $\begin{array}{l}\text { Tiene en cuenta todas las técnicas } \\
\text { de protección posibles y las adapta } \\
\text { al sistema bajo estudio de forma } \\
\text { adecuada }\end{array}$ & $\begin{array}{l}\text { Tiene en cuenta la mayoría de las } \\
\text { técnicas de protección posibles y } \\
\text { las adapta al sistema bajo estudio } \\
\text { de forma adecuada }\end{array}$ & $\begin{array}{l}\text { Tiene en cuenta algunas de las } \\
\text { técnicas de protección posibles y } \\
\text { trata de adaptarlas al sistema bajo } \\
\text { estudio de forma adecuada }\end{array}$ & $\begin{array}{l}\text { No tiene en cuenta las } \\
\text { técnicas de protección posible } \\
\text { y no las adapta al sistema bajo } \\
\text { estudio }\end{array}$ \\
\hline $\begin{array}{l}\text { Explicar y justificar, argumentando adecuadamente, las } \\
\text { opciones elegidas a la hora de resolver problemas reales }\end{array}$ & $\begin{array}{c}\text { Explica y justifica de forma } \\
\text { impecable las opciones elegidas a la } \\
\text { hora de resolver el problema }\end{array}$ & $\begin{array}{c}\text { Explica y justifica de forma } \\
\text { notable las opciones elegidas a la } \\
\text { hora de resolver el problema }\end{array}$ & $\begin{array}{c}\text { Explica y justifica de forma } \\
\text { aceptable las opciones elegidas a } \\
\text { la hora de resolver el problema }\end{array}$ & $\begin{array}{l}\text { No explica ni justifica las } \\
\text { opciones elegidas a la hora de } \\
\text { resolver el problema }\end{array}$ \\
\hline $\begin{array}{l}\text { Explicar de forma sintética, clara y sin ambigüedades } \\
\text { las principales conclusiones alcanzadas tras la } \\
\text { resolución de cada problema }\end{array}$ & $\begin{array}{l}\text { Explica de forma clara y sin } \\
\text { ambigüedades las principales } \\
\text { conclusiones alcanzadas }\end{array}$ & $\begin{array}{c}\text { Explica bien las principales } \\
\text { conclusiones alcanzadas pero hay } \\
\text { alguna imprecisión técnica o del } \\
\text { lenguaje }\end{array}$ & $\begin{array}{c}\text { Explica las principales } \\
\text { conclusiones alcanzadas pero hay } \\
\text { importantes imprecisiones técnica } \\
\text { o del lenguaje }\end{array}$ & $\begin{array}{l}\text { No explica las conclusiones } \\
\text { alcanzadas y/o existen } \\
\text { imprecisiones técnica o del } \\
\text { lenguaje inaceptables }\end{array}$ \\
\hline
\end{tabular}

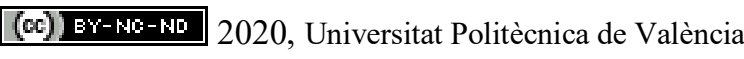

Congreso In-Red (2020) 
Tabla 2. Rúbrica para la actividad de evaluación (2)

\begin{tabular}{|c|c|c|c|c|}
\hline \multirow{3}{*}{ Ítem a evaluar } & \multicolumn{4}{|c|}{ Nivel de competencia } \\
\hline & Excelente & Notable & Suficiente & Insuficiente \\
\hline & 4 & 3 & 2 & 1 \\
\hline Problemática del caso de corrosión presentada & $\begin{array}{c}\text { El caso de corrosión presentado } \\
\text { tiene una problemática asociada } \\
\text { indudable }\end{array}$ & $\begin{array}{l}\text { El caso de corrosión presentado } \\
\text { tiene una problemática asociada } \\
\text { conveniente }\end{array}$ & $\begin{array}{c}\text { El caso de corrosión presentado } \\
\text { tiene una problemática asociada } \\
\text { aceptable }\end{array}$ & $\begin{array}{c}\text { El caso de corrosión } \\
\text { presentado no tiene una } \\
\text { problemática asociada real }\end{array}$ \\
\hline Exposición del problema durante la presentación & $\begin{array}{c}\text { Durante la presentación, el } \\
\text { problema se expone de una manera } \\
\text { clara y muy adecuada }\end{array}$ & $\begin{array}{c}\text { Durante la presentación, el } \\
\text { problema se expone de una manera } \\
\text { adecuada }\end{array}$ & $\begin{array}{c}\text { Durante la presentación, el } \\
\text { problema se expone de una } \\
\text { manera poco clara }\end{array}$ & $\begin{array}{l}\text { Durante la presentación, el } \\
\text { problema se expone de una } \\
\text { manera muy confusa }\end{array}$ \\
\hline Calidad de la presentación oral & $\begin{array}{c}\text { La presentación se realiza de forma } \\
\text { fluida durante todo el tiempo y se } \\
\text { transmite correctamente la } \\
\text { información }\end{array}$ & $\begin{array}{l}\text { La presentación se realiza de } \\
\text { forma fluida la mayor parte del } \\
\text { tiempo y se transmite } \\
\text { correctamente la información }\end{array}$ & $\begin{array}{l}\text { La presentación no se realiza de } \\
\text { forma fluida y se transmite la } \\
\text { información de forma aceptable }\end{array}$ & $\begin{array}{c}\text { La presentación no se realiza } \\
\text { de forma fluida y se transmite } \\
\text { la información de forma } \\
\text { desordenada }\end{array}$ \\
\hline Documentación del caso de corrosión (imágenes, etc.) & $\begin{array}{l}\text { El problema de corrosión está } \\
\text { documentado de forma impecable }\end{array}$ & $\begin{array}{c}\text { El problema de corrosión está bien } \\
\text { documentado }\end{array}$ & $\begin{array}{c}\text { El problema de corrosión está } \\
\text { documentado de forma aceptable, } \\
\text { pero falta material }\end{array}$ & $\begin{array}{l}\text { El problema de corrosión no } \\
\text { está bien documentado }\end{array}$ \\
\hline Lenguaje empleado durante la discusión del problema & $\begin{array}{l}\text { La discusión del problema durante } \\
\text { la presentación se hace con lenguaje } \\
\text { técnico y riguroso }\end{array}$ & $\begin{array}{l}\text { La discusión del problema durante } \\
\text { la presentación se hace en su } \\
\text { mayor parte con lenguaje técnico y } \\
\text { riguroso }\end{array}$ & $\begin{array}{c}\text { La discusión del problema durante } \\
\text { la presentación se hace en } \\
\text { ocasiones con lenguaje técnico y } \\
\text { riguroso }\end{array}$ & $\begin{array}{c}\text { La discusión del problema } \\
\text { durante la presentación NO se } \\
\text { hace con lenguaje técnico y } \\
\text { riguroso }\end{array}$ \\
\hline Soluciones planteadas al problema presentado & $\begin{array}{c}\text { Se presentan soluciones muy } \\
\text { adecuadas al problema presentado }\end{array}$ & $\begin{array}{c}\text { Se presentan soluciones adecuadas } \\
\text { al problema presentado }\end{array}$ & $\begin{array}{l}\text { Se presentan soluciones muy } \\
\text { aceptables al problema } \\
\text { presentado, aunque mejorables }\end{array}$ & $\begin{array}{c}\text { NO se presentan soluciones al } \\
\text { problema presentado }\end{array}$ \\
\hline
\end{tabular}




\section{Resultados obtenidos con las rúbricas}

En este apartado se muestran los valores medios de los resultados obtenidos aplicando las dos rúbricas presentadas más arriba. En primer lugar se presenta un gráfico para la evaluación del informe grupal de resolución de los problemas del ABP, para cada uno de los resultados de aprendizaje definidos anteriormente (Figura 1).

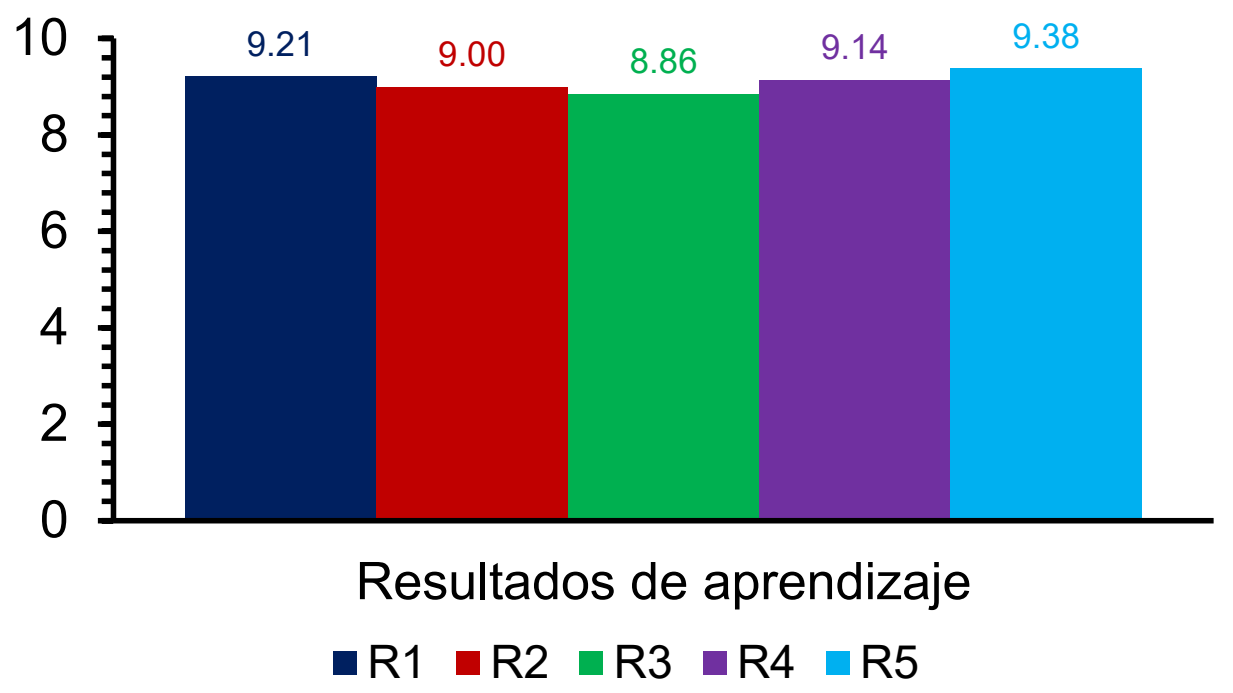

Fig. 1 Calificaciones medias obtenidas para cada uno de los resultados de aprendizaje descritos en la Rúbrica para la actividad de evaluación (1) (Tabla 1).

Como puede verse en el gráfico anterior, las calificaciones medias obtenidas para cada una de los cinco resultados de aprendizaje evaluados son bastante altas, superiores a 8,5 puntos en todos los casos. Además, las desviaciones típicas fueron inferiores a 0,5 puntos en todos los resultados de aprendizaje. De acuerdo con estos resultados, el alumnado encontró algo más de dificultad a la hora de explicar las diferentes técnicas de protección frente a la corrosión existentes y adaptarlas para casos reales particulares (resultado de aprendizaje R3), a pesar de que las calificaciones obtenidas fueron altas en general. Por otro lado, el alumnado destacó especialmente en el resultado de aprendizaje R5, esto es, en explicar de forma sintética, clara y sin ambigüedades las principales conclusiones alcanzadas tras la resolución de cada problema. La conclusión general puede ser que las calificaciones elevadas obtenidas permiten afirmar que, de forma global, se alcanzaron todos los resultados de aprendizaje de forma muy satisfactoria y que, debido a la baja dispersión encontrada en las calificaciones, que estos buenos resultados se dieron para la totalidad de estudiantes. Por tanto, se puede concluir que el método de evaluación mediante la rúbrica de la Tabla 1 es sólido y efectivo.

En la Figura 2 se muestra, para la rúbrica de la Tabla 2 empleada para la actividad de evaluación (2), esto es, para las exposiciones orales tras la práctica de campo, una comparativa entre los resultados medios (para todo el alumnado y para todos los ítems a evaluar) obtenidos cuando la evaluación la hizo el profesorado, cuando la hicieron las y los compañeros (coevaluación) y cuando la hicieron los individuos integrantes de cada grupo (autoevaluación). 


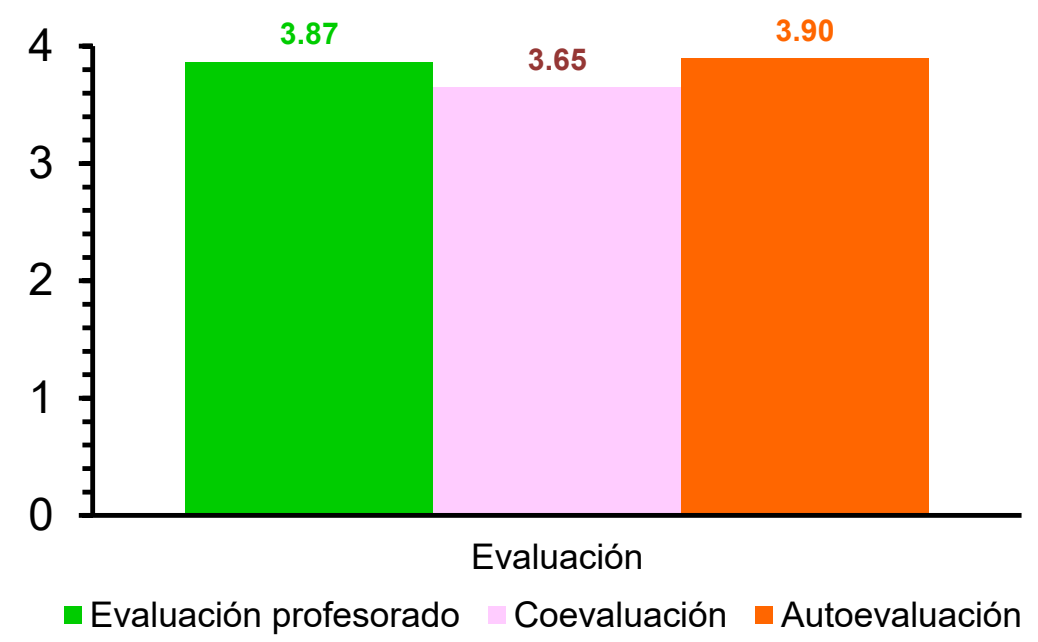

Fig. 2 Comparación entre las calificaciones medias (para todo el alumnado y para todos los items a evaluar) obtenidas cuando la evaluación la hizo el profesorado, cuando la hicieron las y los compañeros (coevaluación) y cuando la hicieron los individuos integrantes de cada grupo (autoevaluación), usando la Rúbrica para la actividad de evaluación (2) (Tabla 2).

De la figura anterior se pueden extraer varias conclusiones. La primera de ellas es que los resultados medios obtenidos para todo el alumnado y para todos los ítems a evaluar de la Tabla 2 son muy satisfactorios, en general. Igual que en el caso anterior, las desviaciones estándar son muy bajas, en este caso inferiores a 0,35 puntos en los tres casos. La segunda conclusión es que el alumnado tiende a ser más crítico con el trabajo presentado por sus semejantes que con su propio trabajo, y también más crítico que el profesorado. Este hecho también pudo comprobarse en los pequeños debates que tuvieron lugar tras cada exposición oral. No obstante, las calificaciones son altas en todos los casos, lo que indica que los trabajos realizados fueron de calidad y que las exposiciones orales se realizaron de forma muy adecuada. La tercera conclusión es que, a la vista de los resultados obtenidos y de la semejanza entre los tipos de evaluación, se puede decir que la rúbrica de la Tabla 2 es apropiada para realizar la evaluación de la actividad (2) descrita más arriba.

\section{Conclusiones}

A la vista de los resultados obtenidos, se puede concluir que el alumnado ha alcanzado con holgura los resultados de aprendizaje, desarrollando así unas competencias clave para su futuro como ingenieros.

En el tema que nos ocupa de corrosión, el alumnado encontró algo más de dificultad a la hora de adaptar las diferentes técnicas de protección frente a la corrosión a los casos reales particulares, lo que puede deberse a la poca práctica que tienen a la hora de enfrentarse a problemas reales (era la primera vez que se enfrentaban a este tipo de metodología activa).

En el caso de las exposiciones orales, el alumnado obtuvo calificaciones elevadas en todos los casos, y se observó una tendencia mayor a la crítica cuando se trataba del trabajo de las y los compañeros que cuando se trataba del trabajo propio.

En general, las rúbricas empleadas para evaluar las diferentes actividades que han surgido de la implementación de la metodología activa del Aprendizaje Basado en Problemas han demostrado ser sólidas y efectivas. 
Agradecimientos: los autores quieren agradecer al Proyecto de Innovación y Mejora Educativa (PIME): Aprendizaje Basado en Problemas para su aplicación en las áreas de Ingeniería Química y de Materiales (B75/18), al ICE y al VECA de la Universitat Politècnica de València.

\section{Referencias}

AUSÍN, V. , ABELLA, V. , DELGADO, V. , AND HORTIGÜELA, D. (2016). Aprendizaje basado en proyectos a través de las TIC. Una experiencia de innovación docente desde las aulas universitarias. Formacion Universitaria, 9, 31-38.

KASSEM, M. A. M. (2018). Improving EFL Students' Speaking Proficiency and Motivation: A Hybrid Problem-based Learning Approach. Theory and Practice in Language Studies, 8, 848-859.

NUÑEZ LOPEZ, S. , ÁVILA PALET, J. E. , AND OLIVARES-OLIVARES, S.-L. (2017). El desarrollo del pensamiento crítico en estudiantes universitarios por medio del Aprendizaje Basado en Problemas. Revista iberoamericana de educación superior, 8, 84-103.

SHAW, S. , LACEY, J. , LEIGHTON, B. , AND WARNER, B. (2006). How problem-based learning supports continuing professional development. Pharmaceutical Journal, 277, 254-255.

TOLEDO MORALES, P. y SÁNCHEZ GARCÍA, J. M. (2018). Aprendizaje Basado En Proyectos: Una Experiencia Universitaria. Profesorado, Revista de Currículum y Formación del Profesorado, 22, 471491.

WYNESS, L. y DALTON, F. (2018). The value of problem-based learning in learning for sustainability: Undergraduate accounting student perspectives. Journal of Accounting Education, 45, 1-19. 